\title{
Generalized Sampling Theorem for Bandpass Signals
}

\author{
Ales Prokes \\ Department of Radio Electronics, Brno University of Technology, Purkynova 118, 61200 Brno, Czech Republic
}

Received 29 September 2005; Revised 19 January 2006; Accepted 26 February 2006

Recommended for Publication by Yuan-Pei Lin

\begin{abstract}
The reconstruction of an unknown continuously defined function $f(t)$ from the samples of the responses of $m$ linear timeinvariant (LTI) systems sampled by the $1 / m$ th Nyquist rate is the aim of the generalized sampling. Papoulis (1977) provided an elegant solution for the case where $f(t)$ is a band-limited function with finite energy and the sampling rate is equal to $2 / m$ times cutoff frequency. In this paper, the scope of the Papoulis theory is extended to the case of bandpass signals. In the first part, a generalized sampling theorem (GST) for bandpass signals is presented. The second part deals with utilizing this theorem for signal recovery from nonuniform samples, and an efficient way of computing images of reconstructing functions for signal recovery is discussed.
\end{abstract}

Copyright () 2006 Hindawi Publishing Corporation. All rights reserved.

\section{INTRODUCTION}

A multichannel sampling involves passing the signal through distinct transformations before sampling. Typical cases of these transformations treated in many works are delays [26] or differentiations of various orders [7]. A generalization of both these cases on the assumption that the signal is represented by a band-limited time-continuous real function $f(t)$ with finite energy was introduced in [1] and developed in [8].

Under certain restrictions mentioned below, a similar generalization can be formed for bandpass signals represented by a function $f(t)$ whose spectrum $F(\omega)$ is assumed to be zero outside the bands $\left(-\omega_{U},-\omega_{L}\right)$ and $\left(\omega_{L}, \omega_{U}\right)$ as depicted in Figure 1(a) while its other properties are identical as in the case of bandpass signals.

If a signal is undersampled (i.e., a higher sampling order is used), then its original spectrum components and their replicas overlap and the frequency intervals $\left(\omega_{L}, \omega_{U}\right)$ and $\left(-\omega_{U}, \omega_{L}\right)$ are divided into several subbands, whose number depends for given frequencies $\omega_{U}$ and $\omega_{L}$ on the sampling frequency $\omega_{S},[4,9]$.

For the introduction of GST, the number of overlapped spectrum replicas has to agree with the sampling order $m$, with the number of subbands inside the frequency ranges $\left(\omega_{L}, \omega_{U}\right)$ and $\left(-\omega_{U},-\omega_{L}\right)$, and with the number of linear systems. As presented in [9], to meet the above demands, $m$ must be an even number and the sampling frequency $\omega_{S}=2 \pi / T_{S}$, where $T_{S}$ is the sampling period, and bandwidth
$\omega_{B}=\omega_{U}-\omega_{L}$ has to meet the following conditions:

$$
\frac{\omega_{L}}{\omega_{U}-\omega_{L}}=\frac{\omega_{L}}{\omega_{B}}=\frac{k_{0}}{m},
$$

where $k_{0}$ is any positive integer number, and

$$
\frac{\omega_{S}}{\omega_{B}}=\frac{2}{m}
$$

An example of a fourth-order sampled signal spectrum in the vicinity of positive and negative original spectral components, if conditions (1) and (2) are fulfilled, is shown in Figure 1(b) and Figure 1(c).

Figure 2 shows a graphical interpretation of the sampling orders $m=2$ to 6 in the plane $\omega_{S} / \omega_{B}$ versus $\omega_{C} / \omega_{B}$, where $\omega_{C}=\left(\omega_{U}+\omega_{L}\right) / 2$, if the common case of bandpass signal sampling is assumed (i.e., frequency $\omega_{S}$ for given $\omega_{B}$ and $\omega_{C}$ is chosen arbitrary) $[4,9]$. Odd orders correspond to the grey areas, whereas even orders correspond to the white ones. The solutions of (1) and (2) for $k_{0}=0,1,2, \ldots$ when $m=2$ and $m=4$ are marked by black points.

\section{GENERALIZED SAMPLING THEOREM FOR BANDPASS SIGNALS}

This expansion deals with the configuration shown in Figure 3. Function $f(t)$ is led into $m$ LTI prefilters (channels) with 


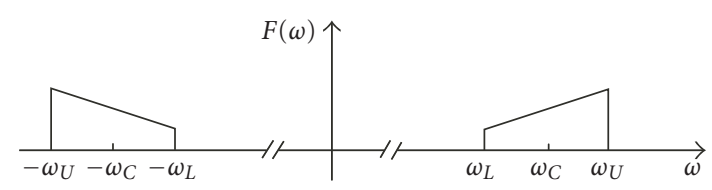

(a)

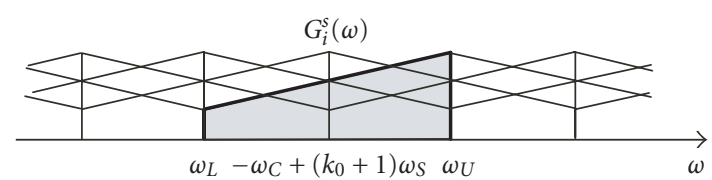

(b)

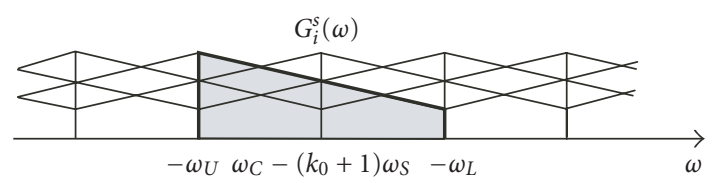

(c)

FIgURE 1: (a) Spectrum of bandpass signal, spectrum of sampled responses $g_{i}(t)$ at the output of LTI prefilters in the vicinity of (b) positive and (c) negative original spectrum components. system functions

$$
H_{1}(\omega), H_{2}(\omega), \ldots, H_{m}(\omega)
$$

The output functions of all prefilters

$$
\begin{aligned}
g_{i}(t)= & \frac{1}{2 \pi} \int_{-\omega_{U}}^{-\omega_{L}} F(\omega) H_{i}(\omega) e^{j \omega t} d \omega \\
& +\frac{1}{2 \pi} \int_{\omega_{L}}^{\omega_{U}} F(\omega) H_{i}(\omega) e^{j \omega t} d \omega
\end{aligned}
$$

are then sampled at $1 / m$ th Nyquist rate related to the cutoff frequency $\omega_{B}$. If mutual independence of the prefilters is assumed and if no noise is present in the system, function $f(t)$ can be exactly reconstructed from samples $g_{i}\left(n T_{S}\right)$, where $T_{S}=m \pi / \omega_{B}$.

For this purpose, the following system of equations has to be formed:

$$
\mathbf{H Y}=\mathbf{R},
$$

where matrix $\mathbf{H}$ and vectors $\mathbf{Y}$ and $\mathbf{R}$ are of the following form:

$$
\mathbf{H}=\left[\begin{array}{cccc}
H_{1}(\omega), & H_{2}(\omega), & \cdots & H_{m}(\omega) \\
H_{1}\left(\omega+\omega_{S}\right), & H_{2}\left(\omega+\omega_{S}\right), & \cdots & H_{m}\left(\omega+\omega_{S}\right) \\
\vdots & \vdots & \vdots \\
H_{1}\left(\omega+\left(\frac{m}{2}-1\right) \omega_{S}\right), & H_{2}\left(\omega+\left(\frac{m}{2}-1\right) \omega_{S}\right), & \cdots & H_{m}\left(\omega+\left(\frac{m}{2}-1\right) \omega_{S}\right) \\
H_{1}\left(\omega+\left(\frac{m}{2}+k_{0}\right) \omega_{S}\right), & H_{2}\left(\omega+\left(\frac{m}{2}+k_{0}\right) \omega_{S}\right), & \cdots & H_{m}\left(\omega+\left(\frac{m}{2}+k_{0}\right) \omega_{S}\right) \\
H_{1}\left(\omega+\left(\frac{m}{2}+k_{0}+1\right) \omega_{S}\right), & H_{2}\left(\omega+\left(\frac{m}{2}+k_{0}+1\right) \omega_{S}\right), & \cdots & H_{m}\left(\omega+\left(\frac{m}{2}+k_{0}+1\right) \omega_{S}\right) \\
\vdots & \vdots & & \\
H_{1}\left(\omega+\left(k_{0}+m-1\right) \omega_{S}\right), & H_{2}\left(\omega+\left(k_{0}+m-1\right) \omega_{S}\right), & \cdots & H_{m}\left(\omega+\left(k_{0}+m-1\right) \omega_{S}\right)
\end{array}\right]
$$

$$
\mathbf{Y}=\left[\begin{array}{c}
Y_{1}(\omega, t) \\
Y_{2}(\omega, t) \\
\cdots \\
\cdots \\
\cdots \\
\cdots \\
Y_{m-1}(\omega, t) \\
Y_{m}(\omega, t)
\end{array}\right], \quad \mathbf{R}=\left[\begin{array}{c}
1 \\
\exp \left(j \omega_{s} t\right) \\
\vdots \\
\exp \left(j\left(\frac{m}{2}-1\right) \omega_{s} t\right) \\
\exp \left(j\left(\frac{m}{2}+k_{0}\right) \omega_{S} t\right) \\
\exp \left(j\left(\frac{m}{2}+k_{0}+1\right) \omega_{S} t\right) \\
\vdots \\
\exp \left(j\left(k_{0}+m-1\right) \omega_{S} t\right)
\end{array}\right]
$$




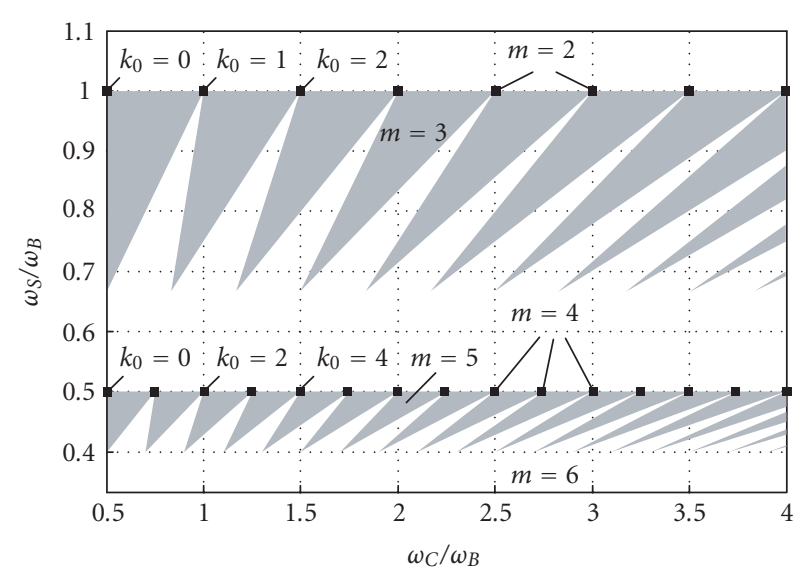

FIGURE 2: Graphical interpretation of the sampling orders.

In the above formulae $t$ is any number and $\omega \in\left(-\omega_{U},-\omega_{U}+\right.$ $\left.\omega_{S}\right)$. This system defines $m$ functions

$$
Y_{i}(\omega, t), Y_{2}(\omega, t), \ldots, Y_{m}(\omega, t)
$$

of $\omega$ and $t$ because the coefficients in matrix $\mathbf{H}$ depend on $\omega$ and the right-hand side depends on $t$. Functions $H_{i}(\omega)$ are on the one hand general, but on the other hand they cannot be entirely arbitrary: they must meet the condition that the determinant of the matrix of coefficients differs from zero for every $\omega \in\left(-\omega_{U},-\omega_{U}+\omega_{S}\right)$.

Since the sampled responses $g_{i}^{s}(t)$ are of the form

$$
g_{i}^{s}(t)=g_{i}(t) \sum_{n=-\infty}^{\infty} \delta\left(t-n T_{s}\right), \quad i=1,2, \ldots, m,
$$

the function $f(t)$ at the output of the multichannel sampling configuration can be described by the following formula:

$$
f(t)=\sum_{i=1}^{m} g_{i}^{s}(t) * y_{i}(t)=\sum_{i=1}^{m} \sum_{n=-\infty}^{\infty} g_{i}\left(n T_{s}\right) y_{i}\left(t-n T_{s}\right),
$$

where

$$
y_{i}(t)=\frac{T_{s}}{2 \pi} \int_{-\omega_{U}}^{-\omega_{U}+\omega_{S}} Y_{i}(\omega, t) e^{j \omega t} d \omega, \quad i=1, \ldots, m
$$

The GST ((5), (10), and (11)) can be proven in a similar way as published in [1].

If we assume that $k_{0}=0$ and put it into (1), then we obtain $\omega_{L}=0$. It means that the bandpass function turns into the band-limited function with cutoff frequency $\omega_{B}$ and the above sampling theorem turns into a generalized sampling expansion [1]. We can say that [1] is a special case of the above GST.

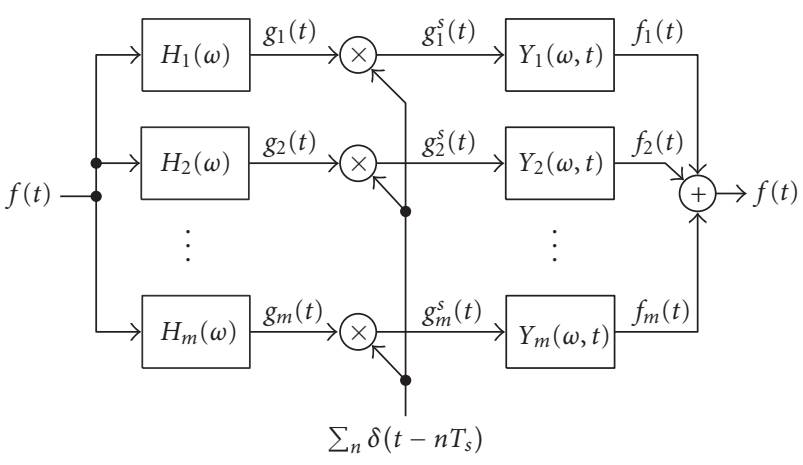

FIGURE 3: Multichannel sampling configuration.

\section{FUNCTION RECOVERY FROM NONUNIFORM SPACED SAMPLES}

As one of the typical applications of the GST, the reconstruction of a signal $f(t)$ from periodically repeated groups of nonuniform spaced samples can be considered [2-5]. It can be obtained if the following formulae hold:

$$
\begin{gathered}
H_{i}(\omega)=e^{j \alpha_{i} \omega}, \\
H_{i}\left(\omega+q \omega_{s}\right)=H_{i}(\omega) e^{j \alpha_{i} q \omega_{s}},
\end{gathered}
$$

where $\alpha_{i}$ denotes time delay in $i$ th branch, that is, the distance between $i$ th sample and the centre of the group.

Substituting (12) into (6), a set of linear equations is obtained, which can be solved by Cramer's rule [10]. For this purpose, $(m+1)$ determinants of the following types have to be solved:

$$
D=\prod_{i=1}^{m} H_{i}(\omega)\left|\begin{array}{cccc}
1 & 1 & \ldots & 1 \\
s_{1}, & s_{2}, & \ldots & s_{m} \\
s_{1}^{2}, & s_{2}^{2}, & \ldots & s_{m}^{2} \\
\vdots & \vdots & \vdots & \vdots \\
s_{1}^{m / 2-1}, & s_{2}^{m / 2-1}, & \ldots & s_{m}^{m / 2-1} \\
s_{1}^{\left(m / 2+k_{0}\right)}, & s_{2}^{\left(m / 2+k_{0}\right)}, & \ldots & s_{m}^{\left(m / 2+k_{0}\right)} \\
s_{1}^{\left(m / 2+k_{0}+1\right)}, & s_{2}^{\left(m / 2+k_{0}+1\right)}, & \ldots & s_{m}^{\left(m / 2+k_{0}+1\right)} \\
\vdots & \vdots & \vdots & \vdots \\
s_{1}^{\left(m / 2+k_{0}-1\right)}, & s_{2}^{\left(m / 2+k_{0}-1\right)} & \ldots & s_{m}^{\left(m / 2+k_{0}-1\right)}
\end{array}\right|,
$$

where $s_{i}=e^{j \alpha_{i} \omega_{s}}, i=1,2, \ldots, m$. Then (11) and consequently (10) are applied to the resulting set of functions $Y(t, \omega)$.

The calculation of (13) using some of the classical methods (e.g., the Laplace expansion [10]) can be difficult for large values of $m$. One of the ways leading under several conditions to a simplification is based on the fact that (13) is of a similar form to Vandermonde's one [10]. However, there is a difference between them, which is hidden in the fact that a jump change in the power for the value of $k_{0}$ appears in the 
lower part of determinant. Finding systematic results, which can be explored for reconstruction, is problematic. A possibly way for $m>2$ and $k_{0} \geq 1$ consists in transformation of (13) into Vandermonde's form by appending the auxiliary terms $\mathbf{X}_{1}, \mathbf{X}_{2}, \mathbf{X}_{3}$, and $\mathbf{S}_{2}$ :

$$
D_{V}=\prod_{i=1}^{m} H_{i}(\omega)\left|\begin{array}{ll}
\mathbf{X}_{1}, & \mathbf{S}_{1} \\
\hline \mathbf{X}_{2}, & \mathbf{S}_{2} \\
\hline \mathbf{X}_{3}, & \mathbf{S}_{3}
\end{array}\right|=\prod_{i=1}^{m} H_{i}(\omega) \triangle,
$$

where

$\triangle$

$$
\begin{array}{|ccc|ccc}
1, & \ldots & 1, & 1, & \ldots & 1 \\
x_{1}, & \ldots & x_{k_{0}}, & S_{1}, & \ldots & S_{m} \\
\vdots & \vdots & \vdots & \vdots & \vdots & \vdots \\
x_{1}^{(m / 2-1)}, & \ldots & x_{k_{0}}^{(m / 2-1)}, & S_{1}^{(m / 2-1)}, & \ldots & S_{m}^{(m / 2-1)} \\
\hline x_{1}^{(m / 2)}, & \ldots & x_{k_{0}}^{(m / 2)}, & S_{1}^{(m / 2)}, & \ldots & S_{m}^{(m / 2)} \\
\vdots & \vdots & \vdots & \vdots & \vdots & \vdots \\
x_{1}^{\left(m / 2+k_{0}-1\right)}, & \ldots & x_{k_{0}}^{\left(m / 2+k_{0}-1\right)} \\
x_{1}^{\left(m / 2+k_{0}\right)}, & \ldots & x_{k_{0}}^{\left(m / 2+k_{0}\right)}, & S_{1}^{\left(m / 2+k_{0}-1\right)}, & \ldots & S_{m}^{\left(m / 2+k_{0}-1\right)} \\
\hline x_{1}^{\left(m / 2+k_{0}+1\right)}, & \ldots & x_{k_{0}}^{\left(m / 2+k_{0}+1\right)}, & S_{1}^{\left(m / 2+k_{0}+1\right)} & \ldots & S_{m}^{\left(m / 2+k_{0}\right)} \\
\vdots & \vdots & \vdots & \vdots & & \vdots \\
x_{1}^{\left(m+k_{0}-1\right)}, & \ldots & x_{k_{0}}^{\left(m+k_{0}-1\right)}
\end{array}
$$

and in using of Vandermonde's rule

$$
\begin{aligned}
D_{V}= & \prod_{i=1}^{m} H_{i}(\omega) \cdot \prod_{i=1}^{m-1} \prod_{j=i+1}^{m}\left(s_{j}-s_{i}\right) \\
& \cdot \prod_{i=1}^{k_{0}} \prod_{j=1}^{m}\left(s_{j}-x_{i}\right) \cdot \prod_{i=1}^{k_{0}-1} \prod_{j=i+1}^{k_{0}}\left(x_{j}-x_{i}\right) .
\end{aligned}
$$

The intermediate term can be rewritten in the following form:

$$
\begin{aligned}
& \left(s_{1}-x_{1}\right) \quad\left(s_{2}-x_{1}\right) \quad \ldots \quad\left(s_{m}-x_{1}\right)
\end{aligned}
$$

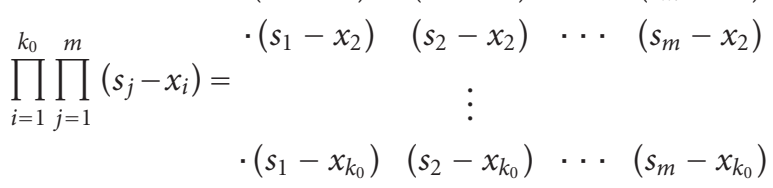

$$
\begin{aligned}
& {\left[x_{1}^{m}-\sigma_{1} x_{1}^{m-1}+\sigma_{2} x_{1}^{m-2}-\cdots+(-1)^{m} \sigma_{m}\right]} \\
& \cdot\left[x_{2}^{m}-\sigma_{1} x_{2}^{m-1}+\sigma_{2} x_{2}^{m-2}-\cdots+(-1)^{m} \sigma_{m}\right] \\
& = \\
& \cdot\left[x_{k_{0}}^{m}-\sigma_{1} x_{k_{0}}^{m-1}+\sigma_{2} x_{k_{0}}^{m-2}-\cdots+(-1)^{m} \sigma_{m}\right] \text {, }
\end{aligned}
$$

where $\sigma_{k}$ are symmetric polynomials consisting of products of all the permutations of $k=1,2, \ldots, m$ terms $s_{1}, s_{2}, \ldots, s_{m}$. That is,

$$
\begin{aligned}
\sigma_{1}= & s_{1}+s_{2}+\cdots+s_{m}, \\
\sigma_{2}= & s_{1} s_{2}+s_{1} s_{3}+\cdots+s_{1} s_{m} \\
& +s_{2} s_{3}+\cdots+s_{2} s_{m}+\cdots+s_{m-1} s_{m},
\end{aligned}
$$

$$
\sigma_{m}=s_{1} s_{2} \cdots s_{m}
$$

Finally, we assume that determinant $\Delta$ in (14) is expanded according to the intermediate band of $\mathbf{X}_{2}, \mathbf{S}_{2}$ using the Laplace expansion. Because the desired determinant (13) (terms $\mathbf{S}_{1}$ and $\boldsymbol{S}_{3}$ ) is an algebraic complement of term $\mathbf{X}_{2}$, it can be revealed as a factor in every multiplication of all the permutations of $k_{0}$ terms of block $\mathbf{X}_{2}$ in the result of (17). Therefore, only one product corresponding to the main diagonal or an adjacent one of the subdeterminant $\mathbf{X}_{2}$ is sufficient. For the final expression of result, special cases of symmetric polynomials have to be defined. They are $\sigma_{0}=1$ and $\sigma_{k}=0$ for $k<0$ and $k>m$.

In this way, determinant (13) is obtained in the form

$$
\begin{aligned}
D= & \prod_{i=1}^{m} H_{i}(\omega) \cdot \prod_{i=1}^{m-1} \prod_{j=i+1}^{m}\left(s_{j}-s_{i}\right) \\
& \cdot(-1)^{k_{0}\left(k_{0}-1\right) / 2}\left|\begin{array}{cccc}
\sigma_{m / 2-k_{0}+1}, & \sigma_{m / 2-k_{0}+2}, & \cdots & \sigma_{m / 2} \\
\sigma_{m / 2-k_{0}+2}, & \sigma_{m / 2-k_{0}+3}, & \cdots & \sigma_{m / 2+1} \\
\vdots & \vdots & \vdots & \vdots \\
\sigma_{m / 2}, & \sigma_{m / 2+1}, & \cdots & \sigma_{m / 2+k_{0}-1}
\end{array}\right| .
\end{aligned}
$$

In a similar way, the determinants $D_{i}, i=1,2, \ldots, m$, which can be formed by replacing the $i$ th column vector with the right-hand side vector $\mathbf{R}$, can be computed. Finally, the desired functions $Y_{i}(\omega, t)$ can be obtained from the ratio $D_{i} / D$.

The determinant in (19) is called the per-symmetric determinant. In the case $k_{0} \geq m$, it contains nonzero terms only near the secondary diagonal.

The efficiency of the described method depends on the values $k_{0}$ and $m$. It is very high in the case $k_{0}<m$, because the order of the determinant that has to be computed is lower than the order of determinant (13), and the resulting expression of (19) contains a large amount of products $\left(s_{j}-s_{i}\right)$, some of which vanish due to divisions $D_{i} / D$. Functions $Y_{i}(\omega, t)$ are then obtained in a very simple form. If $k_{0}$ increases, the determinant order in (19) also increases while the efficiency decreases. In the case $k_{0}>>m$, the order approaches the value $2 k_{0}$. Although the determinant contains the majority of zero terms, the result is more complicated compared to the result of the classical methods of computing the determinants. 


\section{EXAMPLE OF GST APPLICATION}

Although a band-limited function with finite energy is assumed in paragraph 1 , in reality most the signals can be regarded as time-unlimited. A simple example $(m=2)$ of a signal with infinite-energy recovery is shown below. By choosing $H_{1}(\omega)=1$ and $H_{2}(\omega)=e^{j \alpha \omega}$ and substituting them into (10), we obtain

$$
\left[\begin{array}{cc}
1, & e^{j \alpha \omega} \\
1, & e^{j \alpha\left[\omega+\left(k_{0}+1\right) \omega_{s}\right]}
\end{array}\right] \cdot\left[\begin{array}{l}
Y_{1}(\omega, t) \\
Y_{2}(\omega, t)
\end{array}\right]=\left[\begin{array}{c}
1 \\
e^{j \alpha\left(k_{0}+1\right) \omega_{s} t}
\end{array}\right]
$$

The images of reconstructing functions $Y_{1}(\omega, t)$ and $Y_{2}(\omega, t)$ can be found in the form

$$
\begin{aligned}
& Y_{1}(\omega, t)=e^{j\left(\left(k_{0}+1\right) / 2\right) \omega_{s} t} \frac{\sin \left[\left(\left(k_{0}+1\right) / 2\right) \omega_{s}(t-\alpha)\right]}{\sin \left[\left(k_{0}+1\right) \omega_{s} \alpha / 2\right]}, \\
& Y_{2}(\omega, t)=e^{j\left(\left(k_{0}+1\right) / 2\right) \omega_{s} t(t-\alpha)} e^{j \omega \alpha} \frac{\sin \left[\left(k_{0}+1\right) \omega_{s} t / 2\right]}{\sin \left[\left(k_{0}+1\right) \omega_{s} \alpha / 2\right]} .
\end{aligned}
$$

By evaluating (11) under the condition that $\omega_{S}=\omega_{B}$, the reconstructing functions can be expressed as

$$
\begin{aligned}
& y_{1}(t)=-\sin c\left(\frac{\pi t}{T_{s}}\right) \frac{\sin \left[\left(k_{0}+1\right) \pi(t-\alpha) / T_{s}\right]}{\sin \left[\left(k_{0}+1\right) \pi \alpha / T_{s}\right]}, \\
& y_{2}(t)=\sin c\left[\frac{\pi(t-\alpha)}{T_{s}}\right] \frac{\sin \left[\left(k_{0}+1\right) \pi t / T_{s}\right]}{\sin \left[\left(k_{0}+1\right) \pi \alpha / T_{s}\right]},
\end{aligned}
$$

where $\operatorname{sinc}(x)=\sin (x) / x$. The final reconstruction (10) from a limited number of sample groups $n$ can be rewritten in the form

$$
f_{r}(t)=\sum\left[f\left(n T_{s}\right) y_{1}\left(t-n T_{s}\right)+f\left(n T_{s}+\alpha\right) y_{2}\left(t-n T_{s}\right)\right] .
$$

In accordance with (1) and (2), the bandwidth, sampling frequency, carrier frequency, and coefficient $k_{0}$ are chosen as follows: $\omega_{B}=\pi / 2 \mathrm{rad} / \mathrm{s}, \omega_{S}=\pi / 2 \mathrm{rad} / \mathrm{s}, \omega_{C}=2 \pi \mathrm{rad} / \mathrm{s}$, and $k_{0}=7$.

Let function $f(t)$ be given by the formula $f(t)=[1+$ $\left.0.5\left(\sin \omega_{1} t+\sin \omega_{2} t\right)\right] \cos 2 \pi t$. The spectrum of $f(t)$ is then composed of five Dirac pulses at the frequencies $2 \pi \pm \omega_{1}, 2 \pi \pm$ $\omega_{2}$, and $2 \pi$. To demonstrate the reconstruction of a function whose spectrum is inside or partially outside the frequency interval $\left(\omega_{L}, \omega_{U}\right)$, the modulation frequencies of $f(t)$ were chosen as follows: $\omega_{1}=0.2 \mathrm{rad} / \mathrm{s}, \omega_{2}=0.6 \mathrm{rad} / \mathrm{s}$, and $\omega_{2}=$ $0.85 \mathrm{rad} / \mathrm{s}$.

Reconstructing functions $y_{1}(t)$ and $y_{2}(t)$ are shown in Figure 4 . The relation between spectrum $F(\omega)$ and the spectrum of sampled common responses $G_{i}^{s}(\omega)$ is shown in Figure 5 .
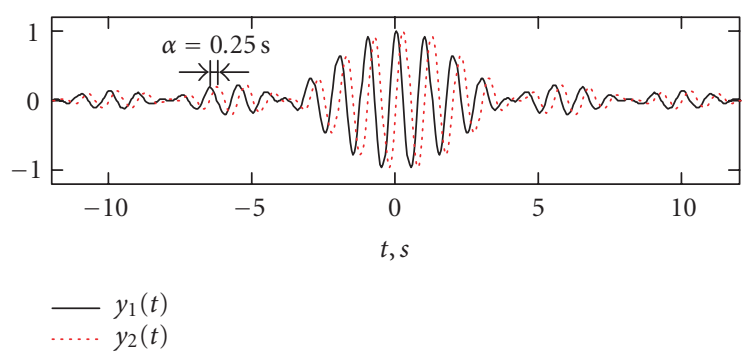

Figure 4: Reconstructing functions $y_{1}(t)$ and $y_{2}(t)$.

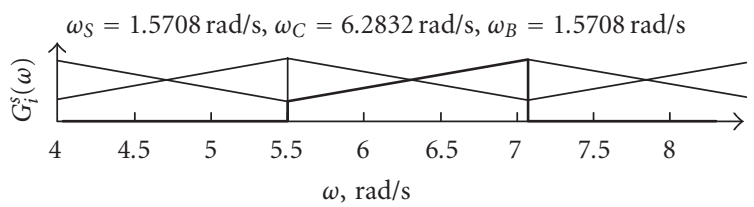

(a)

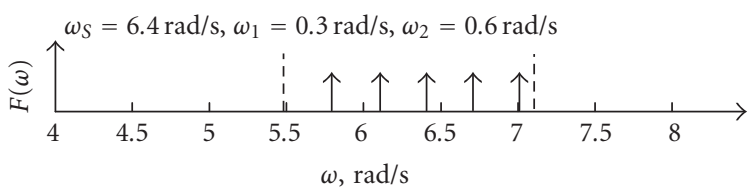

(b)

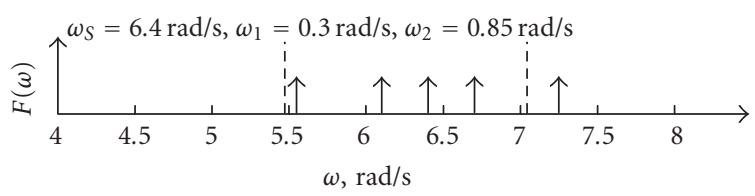

(c)

FIGURE 5: (a) Spectrum of sampled responses in the vicinity of positive original spectrum component for $m=2$. Spectrum of $f(t)$ for both cases of modulation frequencies $\omega_{1}$ and $\omega_{2}(b),(c)$.

Function $f(t)$ and its reconstruction $f_{r}(t)$ from seven groups of samples $n \in[-3,3]$ are plotted in Figure 6 . It is obvious that in the case of an aliasing occurrence Figure 6(b), the reconstruction exhibits a measurable error.

\section{CONCLUSION}

A generalized sampling theorem for time-continuous bandpass signal and the application of this theorem to signal recovery from nonuniform samples have been presented. An efficient method of computing the Fourier images of reconstructing functions for signal recovery from periodically repeated groups of nonuniform spaced samples has then been discussed. As mentioned above, the method presented is suitable for lower values of $k_{0}$ (wideband applications). Finding a simplification similar to (19) in the case $k_{0}>>m$ (narrowband applications) is very difficult and the classical methods of computing the determinant seems to be the best approach. 


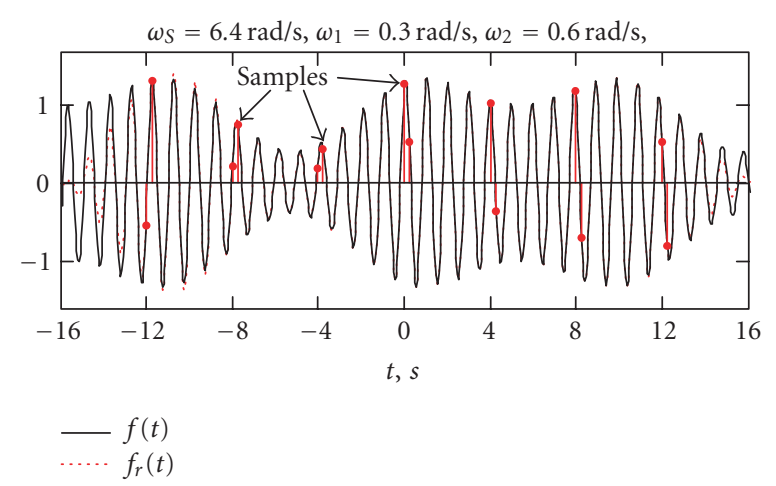

(a)

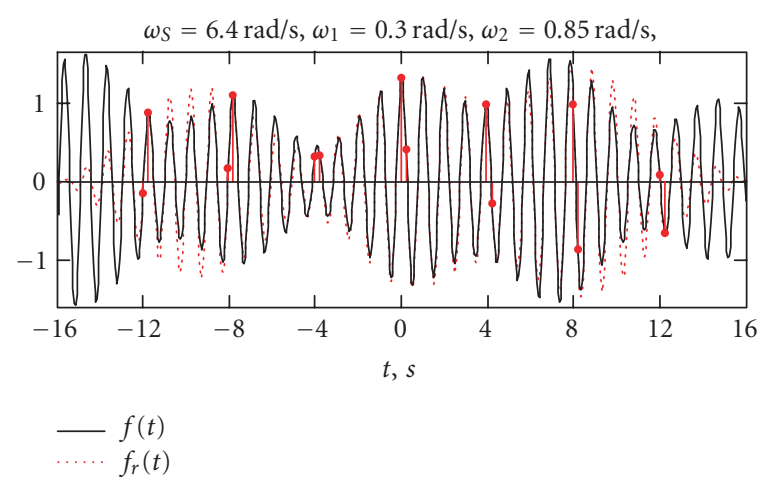

(b)

Figure 6: Function $f(t)$ and its reconstruction $f_{r}(t)$ in cases that spectrum of $f(t)$ is (a) inside or (b) outside frequency interval $\left(\omega_{L}, \omega_{U}\right) .\left(T_{S}=4 \mathrm{~s}, n \in[-3,3], \alpha=0.25 \mathrm{~s}.\right)$

Note that in the case of frequency-limited signal recovery $\left(k_{0}=0\right)$, the determinant of symmetrical polynomials is equal to one and the solution is very simple.

\section{ACKNOWLEDGMENT}

This work has been supported by the Grant GACR (Czech Science Foundation) no. 102/04/0557 "Development of the Digital Wireless Communication Resources," and by the Research Programme MSM0021630513 "Advanced Electronic Communication Systems and Technologies."

\section{REFERENCES}

[1] A. Papoulis, "Generalized sampling expansion," IEEE Transactions on Circuits and Systems, vol. 24, no. 11, pp. 652-654, 1977.

[2] A. Kohlenberg, "Exact interpolation of band-limited functions," Journal of Applied Physics, vol. 24, no. 12, pp. 14321436, 1953.

[3] D. A. Linden, "A discussion of sampling theorems," Proceedings of the IRE, vol. 47, pp. 1219-1226, 1959.

[4] A. J. Coulson, "A generalization of nonuniform bandpass sampling," IEEE Transactions on Signal Processing, vol. 43, no. 3, pp. 694-704, 1995.
[5] Y.-P. Lin and P. P. Vaidyanathan, "Periodically nonuniform sampling of bandpass signals," IEEE Transactions on Circuits and Systems II: Analog and Digital Signal Processing, vol. 45, no. 3, pp. 340-351, 1998.

[6] Y. C. Eldar and A. V. Oppenheim, "Filterbank reconstruction of bandlimited signals from nonuniform and generalized samples," IEEE Transactions on Signal Processing, vol. 48, no. 10, pp. 2864-2875, 2000.

[7] D. A. Linden and N. M. Abramson, "A generalization of the sampling theorem," Information and Control, vol. 3, no. 1, pp. 26-31, 1960.

[8] J. Brown Jr., "Multi-channel sampling of low-pass signals," IEEE Transactions on Circuits and Systems, vol. 28, no. 2, pp. 101-106, 1981.

[9] A. Prokeš, "Parameters determining character of signal spectrum by higher order sampling," in Proceedings of the 8th International Czech-Slovak Scientific Conference (Radioelektronika '98), vol. 2, pp. 376-379, Brno, Czech Republic, June 1998.

[10] C. D. Meyer, Matrix Analysis and Applied Linear Algebra, SIAM, Philadelphia, Pa, USA, 2000.

Ales Prokes was born in Znojmo, Czech Republic, in 1963. He received the M.S. and Ph.D. degrees in electrical engineering from the Brno University of Technology, Czech Republic, in 1988 and 2000, respectively. $\mathrm{He}$ is currently an Assistant Professor at the Brno University of Technology, Department of Radio Electronics. His research interests include nonuniform sampling, velocity measurement based on spatial filter-

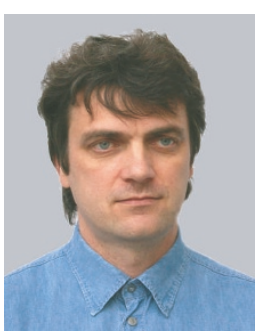
ing and free-space optical communication with emphasis on optical receiver performance analysis and optimization. 